\title{
Identificación molecular de enzimas modificantes de aminoglucósidos en cepas de Enterococcus spp. aisladas en hospitales de la $\mathbf{O}$ ctava Región de Chile
}

Marcela Sepúlveda A ${ }^{1 a}$, Helia Bello ${ }^{2 b}$, Mariana Domínguez $Y^{2 a}$, Sergio Mella $M^{1}$, Raúl Zemelman $Z^{3 c}$, Gerardo González $R^{2 d}$.

Molecular identification of aminoglycosidemodifying enzymes among strains of Enterococcus spp. isolated in hospitals of the VIII Region of Chile

Background: Infectious diseases produced by Enterococcus spp, must be treated with a synergistic combination between a penicillin and an aminoglycoside. High level resistance to aminoglycosides is a serious therapeutic problem, since it predicts the loss of synergistic activity of this antimicrobial combination. Aim: To investigate the presence of genes encoding aminoglycoside-modifying enzymes (AMEs) among strains of Enterococcus spp with high level of resistance to aminoglycosides. Material and methods: The genes encoding some of the AMEs were investigated among 305 aminoglycoside-resistant strains of Enterococcus spp isolated in hospitals of the VIII region of Chile, by dot blot hybridization and Polymerase Chain Reaction (PCR). Results: High level resistance to some aminoglycosides was observed in 104 strains (34.1\%) and 93 of these harbored at least one of the genes encoding the investigated AMEs. Three genes were detected: aac(6')Ieaph(2")Ia (14.8\%) encoding for the enzyme AAC(6')Ie-APH(2")Ia (resistance to all aminoglycosides, except streptomycin); aph(3')IIIa (26\%), and ant(6')Ia (28.5\%) encoding for the phosphorylating enzymes APH( $\left.3^{\prime}\right)$ IIIa (resistance to kanamycin, amikacin and neomycin), and ANT(6')-Ia (resistance only to streptomycin), respectively. None of the strains harbored the gene ant (4') which encode for the enzyme ANT (4'). Conclusion: The low frequency of strains harbouring the bifunctional enzyme $(<15 \%)$, conferring an extended resistance profile to aminoglycosides, allows us to propose the empirical use of aminoglycoside-aminocyclitols, associated to a penicillin, in the treatment of serious infections produced by species of enterococci (Rev Méd Chile 2007; 135: 566-72).

(Key words: Aminoglycosides; Drug resistance, microbial; Enterococcus)

\footnotetext{
Recibido el 27 de julio, 2006. Aceptado el 21 de septiembre, 2006.

Financiamiento: Proyecto 98.036.011-1.0. Dirección de Investigación Universidad de Concepción.

${ }^{1}$ Departamento de Medicina Interna, Facultad de Medicina. ${ }^{2}$ Grupo de Investigación en Resistencia a Antibióticos, Departamento de Microbiología, Facultad de Ciencias Biológicas, Universidad de Concepción y ${ }^{3}$ Facultad de Ciencias y Tecnología, Universidad San Sebastián. Concepción, Chile.

aBioquímica, Magíster en Microbiología

bioquímica, Magíster en Microbiología, Doctor en Ciencias Biológicas

${ }^{\mathrm{c}}$ Químico-Farmacéutico, Dipl. Bacteriol. MSc Public Health

${ }^{d}$ Lic. Biología. Magíster en Microbiología. Doctor en Ciencias Biológicas
}

Correspondencia a: Gerardo González R. Departamento de Microbiología, Facultad de Ciencias Biológicas, Universidad de Concepción. Concepción, Chile. Tel: 41 2203237. Fax: 412245975.

Casilla 160-C. E mail: ggonzal@udec.cl 
$E^{\text {n }}$ terococcus spp presenta resistencia natural a muchos agentes antibacterianos, normalmente activos sobre bacterias Gram positivas, determinando que las infecciones producidas por estos microorganismos sean de difícil tratamiento $^{1}$. El bajo nivel de resistencia a antibióticos aminoglucósidos es una propiedad intrínseca de especies de Enterococci y, a pesar que la causa de esta resistencia no ha sido determinada, probablemente se debería a la ineficiencia de Enterococcus, que posee un metabolismo de tipo anaeróbico, para generar la energía necesaria para que se produzca el transporte del antibiótico a través de la membrana bacteria$\mathrm{na}^{2}$. Por esta razón, el tratamiento de las infecciones causadas por aislados susceptibles de este patógeno se efectúa con la asociación de un antibiótico ß-lactámico y un aminoglucósido habitualmente ampicilina con gentamicina- aprovechando el importante efecto sinérgico que se produce entre estos dos compuestos.

La adquisición de resistencia de alto nivel a aminoglucósidos en Enterococcus spp, es decir, la resistencia que se caracteriza por CIMs $\geq 2000 \mu \mathrm{g} / \mathrm{ml}^{3,4}$ está basada, principalmente, en la síntesis y actividad de enzimas modificantes. Este fenómeno, de gran importancia en los últimos años, caracterizado por altos niveles de resistencia, impide la utilidad clínica de la combinación sinérgica del aminoglicósido con un antibacteriano que actúa a nivel de pared bacteriana ${ }^{5}$.

En la actualidad, Enterococcus spp ha adquirido genes de resistencia a antibióticos aminoglucósidos, que codifican diversas enzimas modificantes de aminoglucósidos, siendo los de mayor importancia clínica:

a. Gen bifuncional aac(6')-Ie-aph(2")-Ia, el cual codifica la enzima bifuncional AAC (6')-IeAPH(2')-Ia. La cepa que posee esta enzima es resistente a todos los aminoglucósidos disponibles con excepción de estreptomicina ${ }^{6}$.

b. Gen aph(3')-IIIa codifica la enzima fosfotransferasa $\mathrm{APH}\left(3^{\prime}\right)$-IIIa, el cual confiere resistencia de alto nivel a kanamicina, amikacina y neomicina ${ }^{7}$.

c. Gen ant(4')-Ia codifica la enzima nucleotidiltransferasa ANT(4")-Ia y que confiere resistencia a tobramicina, amikacina y kanamicina ${ }^{8}$.

d. Gen ant( $\left.6^{\prime}\right)$-Ia codifica la enzima ANT(6')-Ia que confiere resistencia sólo a estreptomicina, siendo el resto de los aminoglucósidos de utilidad clínica?
En este trabajo se determinó la presencia y tipo de EMAs en 305 cepas de Enterococcus spp aisladas en hospitales de la VIII Región, a través de hibridación de ADN y reacción en cadena de la polimerasa (RCP).

\section{Material y MÉTOdO}

Cepas bacterianas. Se incluyeron 305 cepas de Enterococcus spp aisladas en hospitales de la VIII Región entre los años 1996 y 1999. La identificación de éstas se realizó de acuerdo al protocolo estandarizado por nuestro grupo ${ }^{10}$.

Determinación de altos niveles de resistencia a aminoglucósidos. Los altos niveles de resistencia a los antibióticos aminoglucósidos fueron determinados por el método de dilución seriada en $\operatorname{agar}^{11}$ a los siguientes antibióticos aminoglucósidos: gentamicina, estreptomicina, amikacina, kanamicina, neomicina, tobramicina y espectinomicina, en rangos de concentración de $4-2.048 \mu \mathrm{g} / \mathrm{ml}$, y confirmadas en placas de agar BHI (Difco) adicionadas de gentamicina (Sigma-USA) $(500 \mu \mathrm{g} / \mathrm{ml})$, estreptomicina (Sigma-USA) $(2.000 \mu \mathrm{g} / \mathrm{ml})$, amikacina (Sigma-USA) $(2.000 \mu \mathrm{g} / \mathrm{ml})$, kanamicina (Gibco-BRL) $(2.000 \mu \mathrm{g} / \mathrm{ml})$, neomicina (Merck) $(2.000 \mu \mathrm{g} / \mathrm{ml})$, tobramicina (Sigma-USA) $(2.000 \mu \mathrm{g} / \mathrm{ml})^{12}$.

Detección de enzimas modificantes de aminoglucósidos. La presencia y tipo de enzimas modificantes de antibióticos aminoglucósidos fue deducida analizando el fenotipo de resistencia a estos compuestos, determinado por los perfiles de resistencia de cada cepa bacteriana a gentamicina, estreptomicina, amikacina, kanamicina, tobramicina, neomicina y espectinomicina ${ }^{13,14}$. La confirmación de la presencia de las enzimas deducidas anteriormente, fue realizada por detección de los genes que codifican para ellas, utilizando hibridación de $\mathrm{ADN}$ y reacción en cadena de la polimerasa (RCP), de acuerdo a los protocolos modificados de Shaw y col. y Swenson y coll $^{14,15}$, respectivamente. Las secuencias nucleotídicas de los partidores utilizados se muestran en la Tabla 1.

a. Hibridación de ADN. Las membranas de hibridación fueron preparadas depositando en su superficie extractos de ADN de las cepas de Enterococcus spp obtenidas a partir de un 
Tabla 1. Secuencias nucleotídicas de partidores para identificar enzimas modificantes de aminoglucósidos (EM As)

\begin{tabular}{|c|c|c|}
\hline EMAs & $\begin{array}{c}\text { Tamaño } \\
\text { Amplicón (pb) }\end{array}$ & Secuencia de partidores ( $5^{\prime}$ a $3^{\prime}$ ) \\
\hline AAC(6')Ie-APH(2')Ia & 1.375 & $\begin{array}{l}\text { + TGA TGA TTT TCC TTT GAT GT } \\
\text { - CAA TCT TTA TAA GTC CTT TT }\end{array}$ \\
\hline APH(3')IIIa & 682 & $\begin{array}{l}\text { + CCG CTG CGT AAA AGA TAC G } \\
\text { - CTC CCA ATC AGG CTT GAT CC }\end{array}$ \\
\hline ANT(4")Ia & 632 & $\begin{array}{l}\text { + GGA TGA TGT TAA GGC TAT TGG } \\
\text { - GCA CAG ATG GTC ATA ACC TG }\end{array}$ \\
\hline $\operatorname{ANT}\left(6^{\prime}\right) \mathrm{Ia}$ & 577 & $\begin{array}{l}\text { + ACT GGC TTA ATC AAT TTG GG } \\
\text { - GCC TTT CCG CCA CCT CAC CG }\end{array}$ \\
\hline
\end{tabular}

cultivo de 48 h. A $200 \mu \mathrm{l}$ de cultivo se agregó $15 \mu \mathrm{l}$ de lisozima $(7,5 \mathrm{mg} / \mathrm{ml})$, se incubó por 30 min a $37^{\circ} \mathrm{C}$ en agitación constante. Posteriormente, se agregó $200 \mu \mathrm{l}$ de $\mathrm{NaOH}(1 \mathrm{M})$ y con esto se obtuvo una suspensión de células lisadas que liberaron el ADN necesario para el estudio. A partir de este lisado celular, $10 \mu \mathrm{l}$ fueron depositados sobre la membrana y se dejó secar a temperatura ambiente. Para obtener las sondas específicas, se obtuvo productos de RCP generados de la amplificación de las cepas controles utilizando partidores específicos descritos por Swenson y col $^{15}$; Coque y $\mathrm{col}^{16}$; Lemaître y $\mathrm{col}^{17}$. La purificación de los productos de RCP se realizó utilizando el protocolo propuesto en el kit comercial "Concert $^{\mathrm{TM}}$ Rapid PCR Purification System" (GIBCO BRL). Los fragmentos de ADN fueron marcados con fluoresceína-11-dUTP utilizando el kit comercial ECL $^{\mathrm{TM}}$ Random Prime Labelling and Detection System, Version II" (Amersham Pharmacia Biotech Life Science UK Ltd., UK). Finalmente, la hibridación se realizó durante toda la noche a $65^{\circ} \mathrm{C}$ en agitación constante.

b. Reacción en cadena de la polimerasa. De acuerdo a los resultados obtenidos con la hibridación de membranas, es decir, hibridación positiva o negativa con las distintas sondas utilizadas, se determinó en forma presuntiva la presencia o ausencia de las distintas enzimas modificantes de aminoglucósidos. Para la comprobación de estos resultados, se realizó RCP. Brevemente, cuatro a cinco colonias de un cultivo de toda la noche fueron suspendidas en $200 \mu \mathrm{l}$ de agua destilada estéril, posterior a un pulso de centrífuga, $5 \mu \mathrm{l}$ de esta suspensión que contiene el ADN celular, fue utilizado en la mezcla de reacción compuesta por: $1 \mu \mathrm{l}$ de $\mathrm{MgCl} 250 \mathrm{mM}$ (Invitrogen), 2,5 $\mu \mathrm{l}$ dNTPs $1,25 \mathrm{mM}$ (Invitrogen), 2,0 $\mu \mathrm{l}$ de cada uno de los partidores $20 \mathrm{pmol}$ (Invitrogen), 2,5 $\mu$ l de tampón PCR 10X (Invitrogen), 0,15 $\mu$ de Taq polimerasa 5 $\mathrm{U} / \mu \mathrm{l}$ (Invitrogen) y agua destilada estéril en cantidad suficiente para completar $25 \mu \mathrm{l}$ de reacción. La amplificación fue realizada en un termociclador GenAmp PCR System 2400 (PerkinElmer) utilizando el siguiente programa de amplificación: $95^{\circ} \mathrm{C}$ por $10 \mathrm{~min}, 30$ ciclos de $94^{\circ} \mathrm{C}, 30 \mathrm{~s}$, $58^{\circ} \mathrm{C}, 30$ s y $72^{\circ} \mathrm{C}, 30 \mathrm{~s}$ para finalmente un ciclo de 10 min a $72^{\circ} \mathrm{C}$. Los productos de amplificación fueron separados por electroforesis en gel de agarosa $(1,5 \%)$ y la detección de los fragmentos amplificados se realizó tiñendo las muestras con bromuro de etidio $(0,5 \mu \mathrm{g} / \mathrm{ml})$.

\section{REsultados}

De las 305 cepas de Enterococcus spp incluidas en este trabajo, $104(34,1 \%)$, presentaron altos niveles de resistencia (ANR) a alguno de los antibióticos aminoglucósidos estudiados. Entre las cepas de E faecium se encontró el mayor porcentaje de cepas con ANR a los antibióticos aminoglucósidos, con 65,4\%; mientras que en E faecalis se observó sólo en 30,9\% de las cepas. En especies menos frecuentes, el porcentaje de cepas con ANR alcanzó a 42,9\% (Tabla 2). La frecuencia de ANR a los antibióticos ensayados fue: estreptomicina $(32,4 \%)$, 
Tabla 2. Resistencia de alto nivel a aminoglucósidos en cepas de Enterococcus spp aisladas en hospitales de la VIII región de C hile

\begin{tabular}{|lccccccccc|}
\hline \multirow{2}{*}{ Especies } & \multirow{2}{*}{ № } & \multicolumn{8}{c|}{ № cepas con resistencia de alto nivel a aminoglucósidos (\%) } \\
& cepas & G-500 & S-2000 & A-2000 & K-2000 & T-2000 & N-2000 & E-2000 & Total \\
\hline E faecalis & 272 & 41 & 81 & 31 & 70 & 39 & 64 & 0 & 84 \\
& & $(15,4)$ & $(29,8)$ & $(11,4)$ & $(25,7)$ & $(14,3)$ & $(23,5)$ & $(0)$ & $(30,9)$ \\
E faecium & 26 & 3 & 15 & 3 & 14 & 5 & 13 & 0 & 17 \\
& & $(11,5)$ & $(57,7)$ & $(11,5)$ & $(43,8)$ & $(19,2)$ & $(50,0)$ & $(0)$ & $(65,4)$ \\
Enterococcus spp & 7 & 2 & 3 & 0 & 2 & 1 & 2 & 0 & 3 \\
& & $(28,6)$ & $(42,8)$ & $(0,0)$ & $(28,6)$ & $(14,3)$ & $(28,5)$ & $(0)$ & $(42,9)$ \\
Total & \multirow{2}{*}{305} & 46 & 99 & 34 & 86 & 45 & 79 & 0 & 104 \\
& & $(15,1)$ & $(32,4)$ & $(11,1)$ & $(28,2)$ & $(14,8)$ & $(25,9)$ & $(0)$ & $(34,1)$ \\
\hline
\end{tabular}

G-500: gentamicina $500 \mu \mathrm{g}$; S-2000: estreptomicina $2.000 \mu \mathrm{g}$; A-2000: amikacina $2.000 \mu \mathrm{g}$; K-2000: kanamicina $2.000 \mu \mathrm{g}$; T2000: tobramicina $2.000 \mu \mathrm{g}$; N-2000: neomicina $2.000 \mu \mathrm{g}$; E-2000: espectinomicina $2.000 \mu \mathrm{g}$.

kanamicina $(28,2 \%)$, neomicina $(25,9 \%)$, gentamicina $(15,1 \%)$, tobramicina $(14,8 \%)$ y amikacina $11,1 \%$ (Tabla 2).

De acuerdo a los diferentes patrones de resistencia a antibióticos aminoglucósidos encontrados, se postuló que las enzimas ACC(6')-APH(2") (enzima bifuncional), ANT(6') I (adenilante 6' ) y $\mathrm{APH}\left(3^{\prime}\right)$ III (fosforilante $3^{\prime}$ ) participan en la resistencia a estos agentes antibacterianos en las cepas estudiadas. Los patrones de resistencia más comunes incluyeron estreptomicina, kanamicina y neomicina, el cual estaría mediado por las enzimas ANT(6') I y APH(3') III. Por su parte, el ANR a gentamicina, estreptomicina, kanamicina, amikacina, neomicina y tobramicina sería consecuencia de la síntesis de las enzimas bifuncionales, adenilante 6 ' y fosforilante 3 '.

Los resultados de la hibridación con sondas específicas para las EMAs, en las cepas de Enterococcus spp con altos niveles de resistencia a antibióticos aminoglucósidos, se presentan en las Figuras 1,2 y 3 , resultados que fueron confirmados mediante amplificación por RCP (Figura 4). Así, por ejemplo, en la cepa 219, que no presenta ANR a antibióticos aminoglucósidos, no se produjo amplificación de los genes investigados (Figura 4, carriles 8, 9, 10 y 11). Por otra parte, la cepa 88, que fenotípicamente evidenciaba la posibilidad de poseer las cuatro enzimas en estudio, resultó positiva para AAC(6') Ie-APH(2")Ia (posición C2, Figura 1), ANT(6')Ia (posición C2, Figura 2) y APH(3')IIIa (posición C2, Figura 3). Mediante la amplificación genética se confirmó la presencia de estas enzimas y se descartó la presencia de ANT(4')Ia (Figura 4, carriles 4, 5, 6 y 7). Finalmente, el patrón de resistencia que presentó la cepa 132 sugirió la presencia de las enzimas AAC(6')IeAPH(2")Ia, ANT(6')Ia y APH(3')IIIa, lo que fue comprobado por hibridación en colonia (Posición C11, Figuras 1, 2 y 3) y posteriormente por RCP, al obtener los productos de amplificación de 1.375 pb, 577 pb y 682 pb, correspondientes a un fragmento intragénico de las enzimas $\mathrm{AAC}\left(6^{\prime}\right) \mathrm{Ie}-$ APH(2")Ia, ANT(6')Ia y APH(3')IIIa, respectivamente (Figura 4, carriles 12, 13, 14 y 15). En ninguna de las cepas estudiadas se obtuvo amplificación para el gen que codifica la enzima ANT(4')Ia. En la Figura 5 se resume las enzimas que fueron detectadas en las cepas de Enterococcus spp estudiadas.

\section{DisCUSIÓN}

Los altos niveles de resistencia de aislados de enterococos frente a aminoglucósidos se debe, principalmente, a la presencia de enzimas modificantes de aminoglucósidos (EMA's), hecho de relevancia clínica, ya que predice la pérdida de sinergia entre un aminoglucósido y un $\beta_{\text {-lactámico }}{ }^{18}$. De las cepas aisladas en la región del Bío-Bío, 104 presentaron altos niveles de resistencia a algún aminoglucósido. Estreptomicina, kanamicina y neomicina 
presentaron la menor actividad en las cepas estudiadas. La resistencia de alto nivel a gentamicina fue menos frecuente y estos valores son concordantes con los obtenidos en la investigación realizada por el programa SENTRY en América Latina en los últimos años ${ }^{19}$. De acuerdo a los patrones de resistencia presentados por las cepas de Enterococcus spp con ANR, se postuló que las enzimas ACC(6')-IeAPH(2")-Ia, ANT(6)-Ia, APH(3')-IIIa y ANT(4')-Ia participaban en esta resistencia. Para poder comprobar la presencia de estas enzimas, se investigaron los genes que codifican cada una de ellas hibridando el ADN de cada cepa con las sondas de las enzimas bifuncionales, adenilante 6'-Ia y fosforilante 3'-IIIa, concluyendo que la presencia de la enzima bifuncional AAC(6')-APH(2"), sola o asociada a APH(3')-IIIa otorga resistencia a todos los aminoglucósidos, excepto a estreptomicina. En cambio, cuando las cepas presentan susceptibilidad a gentamicina, pero resistencia al resto de aminoglucósidos se encontró la presencia de la enzima APH(3')-IIIa, sola o asociada a ANT(6'), y en este último caso también presentaba resistencia a estreptomicina. Por otra parte, en aquellas cepas que mostraron resistencia a todos los aminoglucósidos estaban presentes las tres enzimas estudiadas.

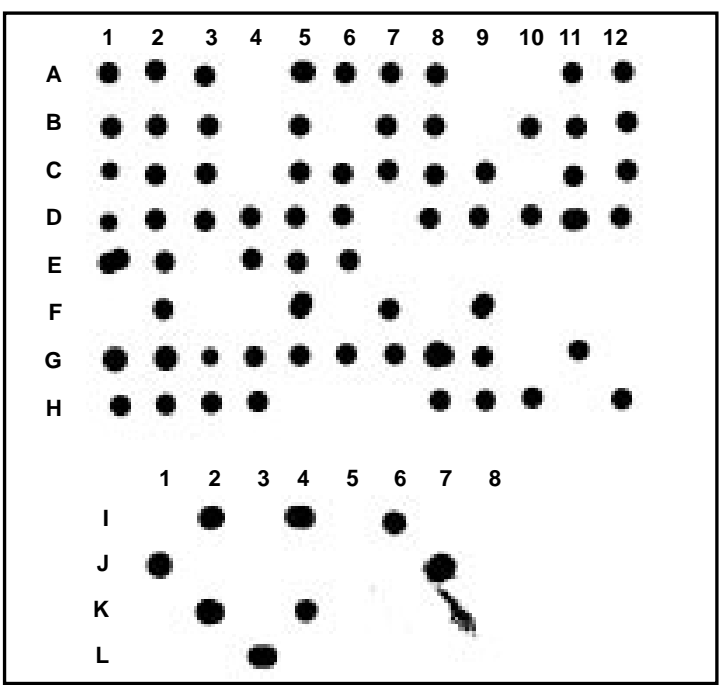

Figura 2. Hibridación con sonda para la enzima ANT(6')Ia. En las posiciones H11 y L1 se ubica el control negativo E faecalis ATCC 29212, en la posición H12 y L3 se ubica el control positivo, cepa E faecalis ATCC 51299. En la posición F1 se ubica la cepa clínica 219 control negativo. En resto de posiciones se ubican cepas clínicas que presentan ANR a algún antibiótico aminoglicósido.

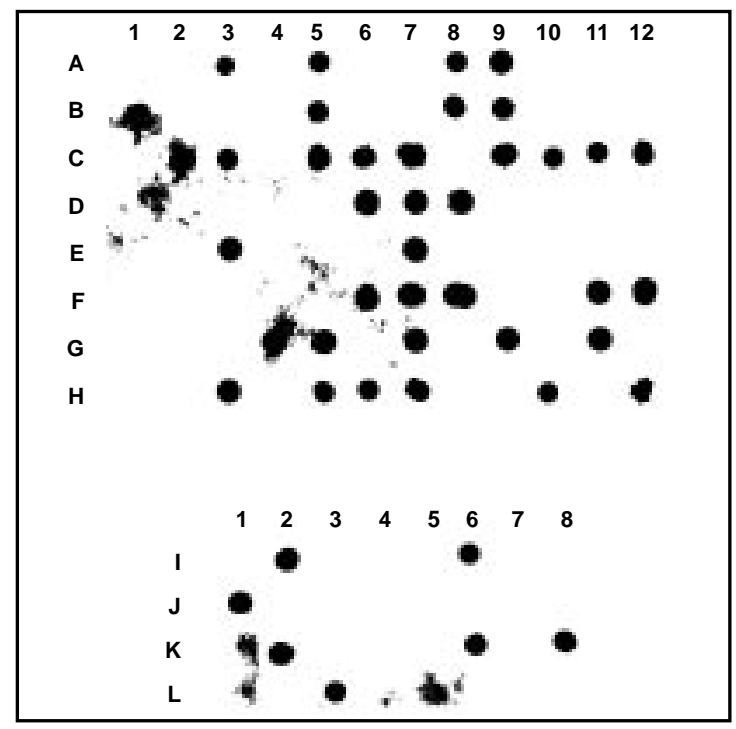

Figura 1. Hibridación con sonda para enzima bifuncional ACC(6')Ie-APH(2")Ia. En las posiciones H11 y L1 se ubica el control negativo E faecalis ATCC 29212, en la posición H12 y L3 se ubica el control positivo, cepa E faecalis ATCC 51299. En la posición F1 se ubica la cepa clínica 219, control negativo. En resto de posiciones se ubican cepas clínicas que presentan ANR a algún antibiótico aminoglucósido.

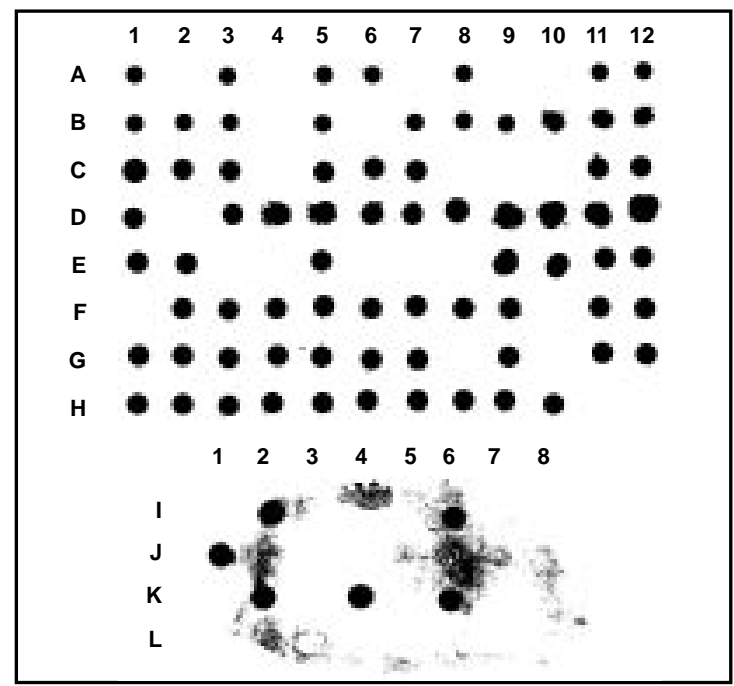

Figura 3. Hibridación con sonda para la enzima APH(3')IIIa. En las posiciones H11 y L1 se ubica el control negativo E faecalis ATCC 29212, en la posición H12 y L3 se ubica la cepa E faecalis ATCC 51299, control negativo. En la posición F1 se ubica la cepa clínica 219 control negativo. En resto de posiciones se ubican cepas clínicas que presentan ANR a algún antibiótico aminoglicósido. 


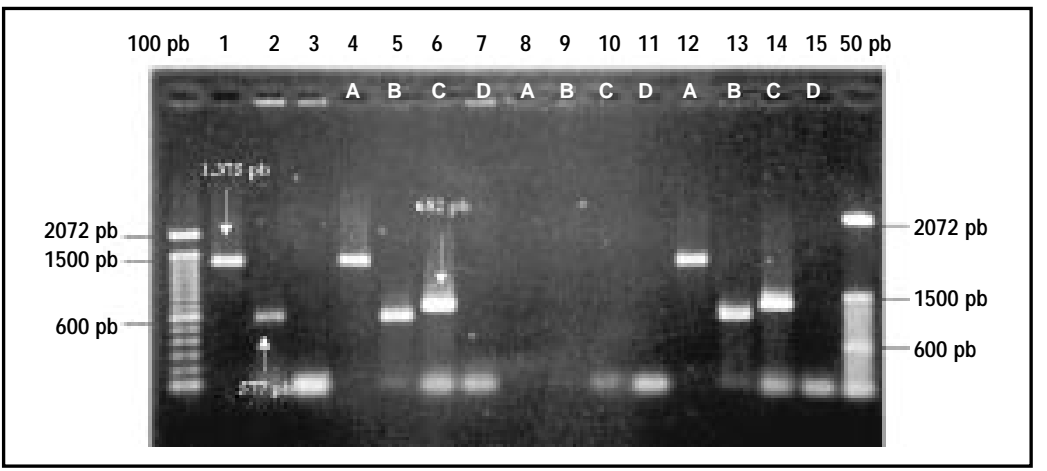

Figura 4. Amplificación por RCP de genes de EMAs con partidores específicos.

1: cepa control gen aac $\left(6^{\prime}\right) \mathrm{Ie}$ aph(2")Ia; 2: cepa control gen ant(6')Ia; 3: control mezcla de reacción; 4, 5, 6 y 7: cepa 88; 8, 9, 10 y 11: cepa 219 (control negativo); 12, 13, 14 y 15: cepa 132. A: $\operatorname{aac}\left(6^{\prime}\right)-\operatorname{aph}\left(2^{\prime \prime}\right) I a ; B$ : ant(6')Ia C: aph(3')IIIa D: ant(4')Ia.

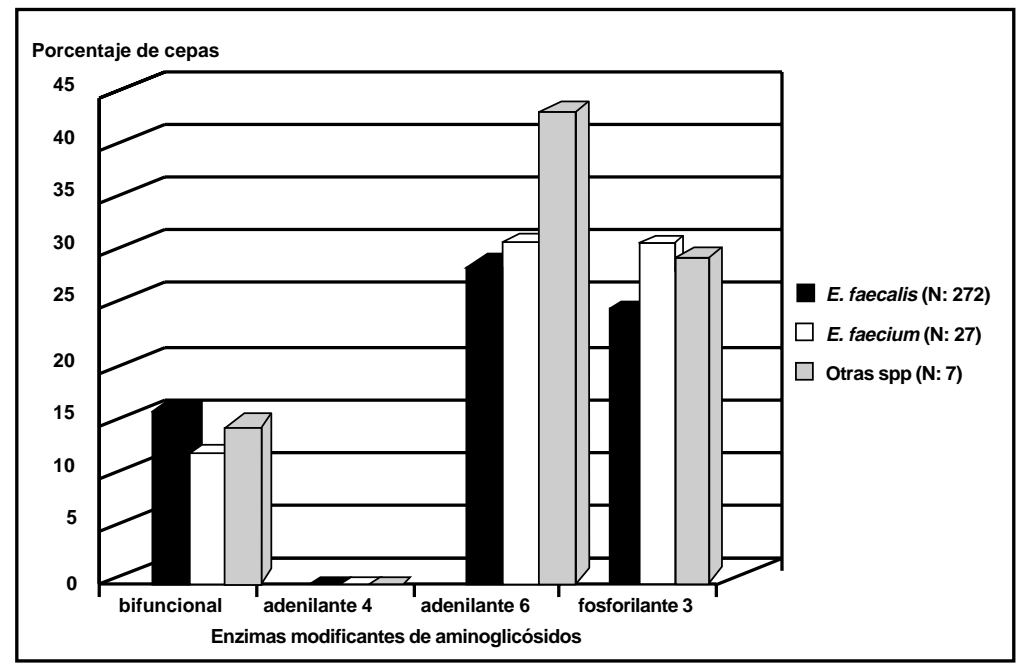

Figura 5. Distribución de enzimas modificantes de aminoglucósidos (EMA) detectadas en cepas de Enterococcus spp aisladas en hospitales de la VIII región.

Se encontró una buena correlación entre los resultados de la hibridación y RCP, lo que además coincidió, en su mayoría, con los patrones fenotípicos de resistencia. La excepción a esta correlación se observó al analizar las cepas con altos niveles de resistencia a estreptomicina, ya que 12 de ellas que fenotípicamente muestran resistencia de alto nivel no hibridaron con la sonda específica. Posterior a la estandarización de la metodología de RCP se puso en evidencia que estas cepas no poseen el gen ant $\left(6^{\prime}\right)$ que codifica la síntesis de la enzima ANT( $\left.6^{\prime}\right)$. Por lo tanto, se postuló que esta resistencia es producto de una mutación a nivel ribosomal, lo que ha sido informado con anterioridad $^{20}$.

La determinación por RCP de los genes ant(4') en las cepas en estudio resultó negativa a pesar que se había determinado presuntamente su presencia según el patrón de resistencia. Esto podría explicarse por la presencia de la enzima bifuncional, que confiere resistencia a la mayoría de los aminoglucósidos, menos a estreptomicina. Por lo tanto, la resistencia a tobramicina puede explicarse por la presencia de la enzima bifuncional y no de la enzima ANT(4')-Ia) ${ }^{13}$.

En nuestra región, y de acuerdo a estos resultados, dada la baja frecuencia de aislados de Enterococcus spp con ANR a amikacina $(11,1 \%)$ y gentamicina (15\%), estos agentes todavía pueden utilizarse en forma empírica, asociándolos a una penicilina, para el tratamiento de infecciones enterocócicas graves en las cuales se requiere de un potente efecto bactericida. 


\section{REFERENCIAS}

1. Shepard BD, Gilmore MS. Antibiotic-resistant enterococci: the mechanisms and dynamic of drug introduction and resistance. Microbes Infect 2002; 4: $215-24$.

2. LeCLeRCQ R. Enterococci acquire new kinds of resistance. Clin Infect Dis 1994; 24 (Suppl 1): 80-4.

3. Moellering R Jr, Wennersten C, Medrek T, Weinberg AN. Prevalence of high-level resistance to aminoglycosides in clinical isolates of enterococci. Antimicrob Agentes Chemother 1970; 1: 335-40.

4. Patterson JE, Zervos MJ. High-level gentamicin resistance in Enterococcus: microbiology, genetic basis, and epidemiology. Rev Infect Dis 1990; 12: 644-52.

5. DAvIs BD. Bactericidal sinergism between 3 lactams and aminoglycosides: mechanism and possible therapeutic implications. Rev Infect Dis 1982; 4: 237-45.

6. Ferreti JJ, Gilmore KS, Courvalin P. Nucleotide sequence analysis of the gene specifying the bifunctional 6'-aminoglycoside acetyltransferase 2'-aminoglycoside phosphotransferase enzyme in Streptococcus faecalis and identification and cloning of gene regions specifying the two activities. J Bacteriol 1986; 167: 631-8.

7. Mckay GA, Roestamadji J, Mobashery S, Wright GD. Recognition of aminoglycoside antibiotics by enterococcal-staphylococcal aminoglycoside 3'phosphotransferase type IIIa: Role of substrate amino groups. Antimicrob Agents Chemother 1996; 40: 2648-50.

8. Carlier C, Courvalin P. Emergence of 4',4"-aminoglycoside nucleotidyltransferase in enterococci. Antimicrob Agents Chemother 1990; 34: 1565-9.

9. Ounissi H, Derlot E, Carlier C, Courvalin P. Gene homogeneity for aminoglycoside-modifying enzymes in Gram-positive cocci. Antimicrob Agents Chemother 1990; 34: 2164-8.

10. Sepúlveda M, Bello H, Ruiz M, Hormazábal J, Domínguez M, González G ET AL. Metodología clásica y molecular en la identificación de especies de Enterococcus spp. Rev Méd Chile 2002; 130: 45-9.

11. National Committee for Clinical Laboratory StanDARDS. 1997. Methods for dilution antimicrobial susceptibility tests for bacteria that grow aerobically. Approved Standard M7-A4. National Commit- tee for Clinical Laboratory Standards, Villanova. PA.

12. National Committe for Clinical Laboratory StanDARDS. 1998. Performance Standards for antimicrobial susceptibility testing. Ninth informational supplement M100-S8. National Committee for Clinical Laboratory Standards. Wayne, Pa.

13. Shaw KJ, Sabatelli FJ, Naples L, Mann P, Hare RS, Miller HG. The application of molecular techniques for the study of aminoglycoside resistance. En Woodford, N. and Johnson, A.P, Eds. Methods in Molecular Medicine, Vol 15: Molecular Bacteriology: Protocols and Clinical Applications. Tatowa, New Jersey: Human Press Inc., 1998; 555-77.

14. Shaw KJ, Hare RS, Sabatelli FJ, Rizzo M, Cramer CA, NAPLes L et AL. Correlation between aminoglycoside resistance profiles and DNA hybridization of clinical isolates. Antimicrob Agents Chemother 1991; 35: 2253-61.

15. Swenson JM, Ferraro MJ, Sham DF, Clark NC, Culver DH, Tenover FC ET AL. Multilaboratory evaluation of screening methods for detection of high-level aminoglycoside resistance in enterococci. J Clin Microbiol 1995; 33: 3008-18.

16. Coque TM, Arduino RC, Murray BE. High-level resistance to aminoglycosides: comparison of community and nosocomial fecal isolates of enterococci. Clin Infect Dis 1995; 20: 1048-51.

17. Lemaître N, Sougakoff W, Masmoudi A, Fievet M-H, Bismuth R, JARLIER V. Characterization of gentamicin-susceptible strains of methicillin-resistant Staphylococcus aureus involved in nosocomial spread. J Clin Microbiol 1998; 36: 81-5.

18. Leclerg R, DutKa-Malen S, Brisson-Noel A, Molinas C, Derlot E, Arthur M et al. Resistance of enterococci to aminoglycosides and glycopeptides. Clin Infect Dis 1992; 15: 495-501.

19. Low DE, Keller N, Barth A, Jones RN. Clinical prevalence, antimicrobial susceptibility, and geographic resistance patterns of enterococci: Results from the SENTRY antimicrobial surveillance program, 1997-1999. Clin Infect Dis 2001; 32 (Suppl 2): $133-45$.

20. Eliopoulos GM, Wennersten C, Zighelboim-Daum S, Reiszner E, Goldmann D, Moellering RC Jr. Highlevel resistance to gentamicin in clinical isolates of Streptococcus (Enterococcus) faecium. Antimicrob Agents Chemother 1988; 32: 1528-32. 